\title{
Semantic Wiki as a Lightweight Knowledge Management System
}

\author{
Hendry Muljadi ${ }^{1}$, Hideaki Takeda ${ }^{1}$, Aman Shakya ${ }^{2}$, Shoko Kawamoto ${ }^{1}$, \\ Satoshi Kobayashi ${ }^{1}$, Asao Fujiyama ${ }^{1}$, and Koichi Ando ${ }^{3}$ \\ ${ }^{1}$ National Institute of Informatics, 2-1-2, Hitotsubashi, Chiyoda-ku, Tokyo, Japan 69121 \\ \{hendry, takeda, skawamot, satoshi-k, afujiyam\}@nii.ac.jp \\ ${ }^{2}$ Asian Institute of Technology, P.O.Box 4, Klong Luang, Pathumthani 12120, \\ aman.shakya@ait.ac.th \\ ${ }^{3}$ Shibaura Institute of Technology, 3-7-5, Toyosu, Koto-ku, Tokyo, Japan \\ andou@sic.shibaura-it.ac.jp
}

\begin{abstract}
Since its birth in 1995, Wiki has become more and more popular. This paper presents a Semantic Wiki, a Wiki extended to include the ideas of Semantic Web. The proposed Semantic Wiki uses a simple Wiki syntax to write labeled links which represent RDF triples. By enabling the writing of labeled links, Semantic Wiki may provide an easy-to-use and flexible environment for an integrated management of content and metadata, so that Semantic Wiki may be used as a lightweight knowledge management system.
\end{abstract}

Keywords: Wiki, MediaWiki, Semantic Wiki, metadata, RDF

\section{Introduction}

Since its birth in 1995, Wiki has become more and more popular. It is a simple publishing system that is easy to learn and quick to use. In Wiki, people can create or edit a Wiki page using a simple syntax to write content [1]. The popularity of Wikipedia ${ }^{1}$, the online encyclopedia, has proven how Wiki is effective for the collaboration on the web. Wiki has been considered for the development of a knowledge management system [2],[3]. It has gained significant attention from industry as well [4].

Nowadays there are so many Wiki engines available. One of the famous Wiki software is MediaWiki ${ }^{2}$, which is being used to run Wikipedia. MediaWiki has the category management function which allows a Wiki page under the namespace (“Category:") to be used as a metadata, and also allows user to create class and subclass relation, as well as class and instance relation between Wiki pages. In other words, MediaWiki has the capability to manage: (1) contents, (2) metadata, and (3) the relations between contents and metadata. However, the metadata is not suitable for processing by applications.

\footnotetext{
1 en.wikipedia.org/wiki/Wikipedia

2 www.mediawiki.org
} 
Nevertheless, in a Wiki environment, it is easy to make an Resource Description Framework (RDF) resource, since a Wiki page always has a URL, e.g. http://hostname/wiki/pagename, and this URL can be used as an URI of an RDF resource. RDF is a language to express metadata that is suitable for processing by applications [5]. It consists of subject-predicate-object triples that state specific facts about resources or concepts, e.g. “[Homer] $<$ HasChild $>$ [Bart]”, where subject, predicate and object (if not a literal) are identified via URIs. Constructing RDF triples in a Wiki environment can be done by enabling the construction of labeled links [6]. The labeled link represents the RDF property that links the RDF subject with its object.

For the development of a lightweight knowledge management system, a Wiki is extended to enable the writing of labeled links. This paper presents MewKISS, a Wiki extended to enable the integrated management of content and metadata. Section 2 presents the extension of Wiki to enable the integrated management of content and metadata. Section 3 shows the implementation cases of MewKISS as a lightweight knowledge management system.

\section{MewKISS}

\subsection{Semantic Extension of Wiki}

MewKISS is an abbreviation for MediaWiki with Simple Semantics. It uses MediaWiki as its basis Wiki engine. By extending MediaWiki, the Semantic Wiki will have the benefit of having all the functions available in MediaWiki as a content management system.

Using the existing category management function as a reference, a new syntax is created to write the labeled links. Wiki syntax to write the labeled link is [[term:target_page|property]]. Each time this syntax is written on a Wiki page, the triple will be stored into the new table in the Wiki database.

Fig. 1 shows the example of the Wiki syntax writing on a Wiki page. The Wiki page on which the syntax is written will become the source page of the RDF triple. Fig.2(a), (b), (c) show how the labeled link relations are displayed on the source page, target page and property page respectively. Displaying labeled link relation allows users to navigate the relation between pages easily.

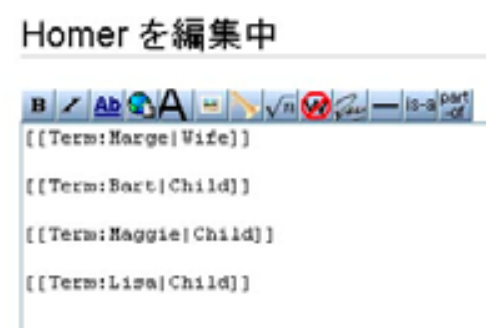

Fig. 1. Using the Wiki syntax for RDF triple construction 
Enabling MediaWiki to write labeled links with simple syntax allows users to create and manage relations between Wiki pages easily and flexibly. The writing of labeled links allows users to write and edit RDF triples even though they have no knowledge about it. Thus, MewKISS can be used as an integrated content and metadata management system.

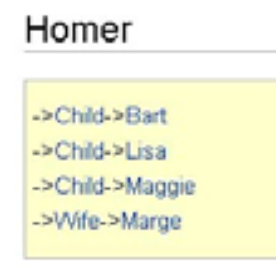

(a) Display of the source_page (b) Display of the target_page ->property->target_page

\section{Bart}

who?

$$
<-C h i l d<-H o m e r
$$

\section{Child}

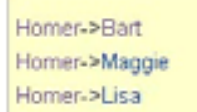

Homer->Lisa

(c) Display of the property page source_page->target_page

Fig. 2. Display on the Wiki pages

\subsection{Mapping to Other Semantic Web Application}

Semantic Wiki has becoming more and more popular. There are more than 20 prototypes available ${ }^{3}$. Surveying the current trend, Semantic Wikis have gone into two poles. The first one emphasizes the need to build a Semantic Wiki as a Semantic Web application. It is useful for domain experts, but will leave non-technical users away from it. The other one emphasizes user-friendliness, especially for non-technical users. It leaves the more technical Semantic Web aspect to other applications.

MewKISS emphasizes the user-friendliness of the Wiki engine. It is developed to allow non-technical users to manage metadata easily, and leaves the more technical aspects to external applications.

Fig.3 shows the overall structure of MewKISS. The RDF triples are stored in a table in the MewKISS database. The stored RDF triples can be exported to RDF database such as Sesame ${ }^{4}$. Using Sesame, users can explore the exported RDF triples (see Fig.4), make queries etc. In other words, MewKISS can be used as a bridge between non-technical users and Semantic Web technology.

\section{Implementation}

The proposed Semantic Wiki is developed for the development of a Web-based Japanese Biodictionary. Currently, it is also used for the development of a manufacturing feature library, a feature-based manufacturing information management system.

\footnotetext{
3 http://www.semwiki.org/

${ }^{4}$ http://openrdf.org/
} 


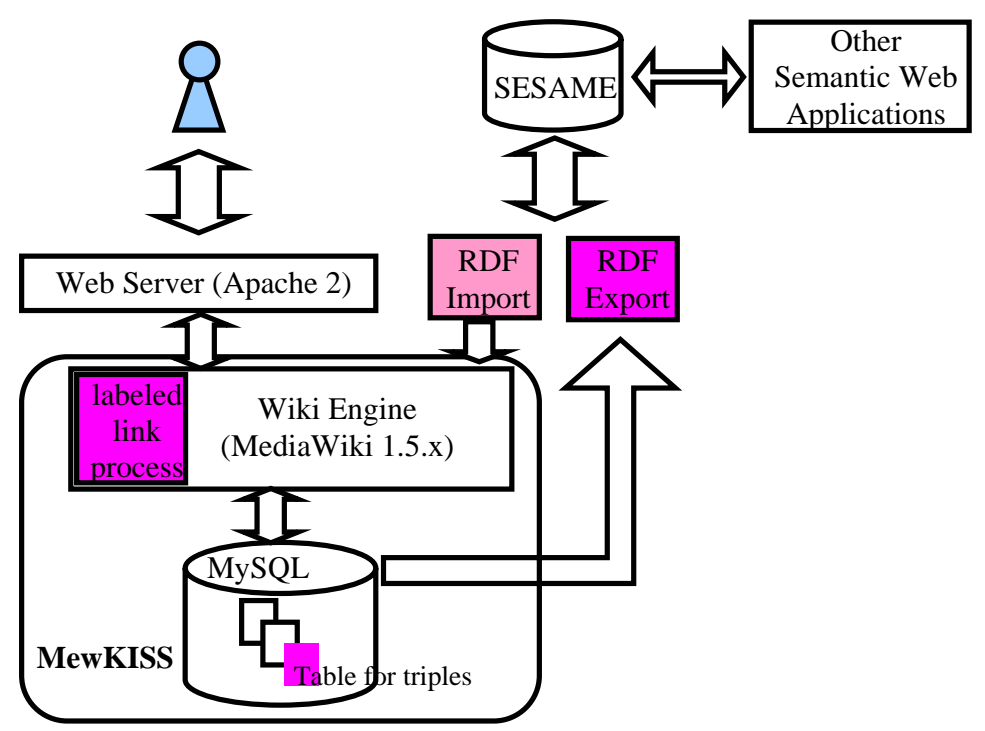

Fig. 3. The overall structure of MewKISS

\section{Explore repository}

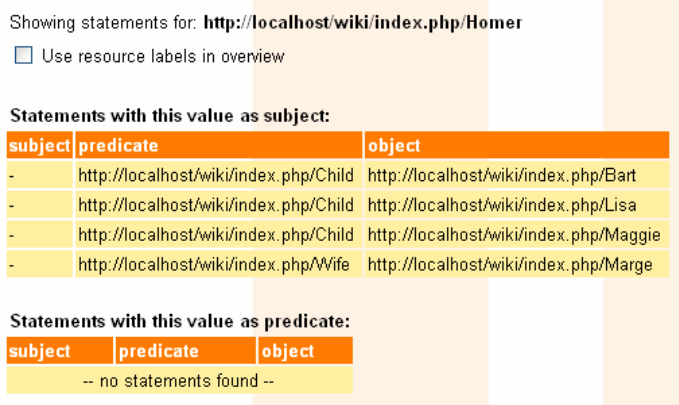

Fig. 4. Exploring the RDF repository

\subsection{Semantic Wiki for the development of a Web-based Japanese Biodictionary}

Developing a Web-based Japanese Biodictionary requires an environment where researchers from various biology fields may collaborate to create and maintain the content and the metadata of the dictionary. Semantic Wiki is able to provide such environment. It also provides the navigation support to manage relation between terms.

Currently, the prototype system contains more than 4,400 terms. Fig. 5 shows the editing page of a Japanese biology term. Users can write and edit the contents and the 
relation between pages easily and visually. In other words, users can write and edit RDF triples even though they have no knowledge about it. Fig. 6 shows the Wiki page of the Japanese biology term. The labeled link relations that are written using the Wiki syntax are displayed. By displaying the labeled link relations, users can navigate the relation between terms visually.

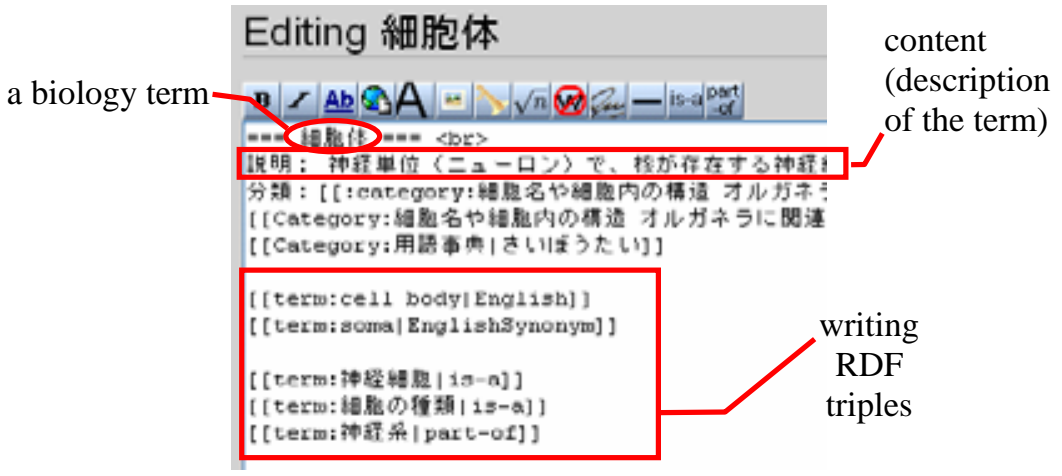

Fig. 5. Editing box of the Wiki page

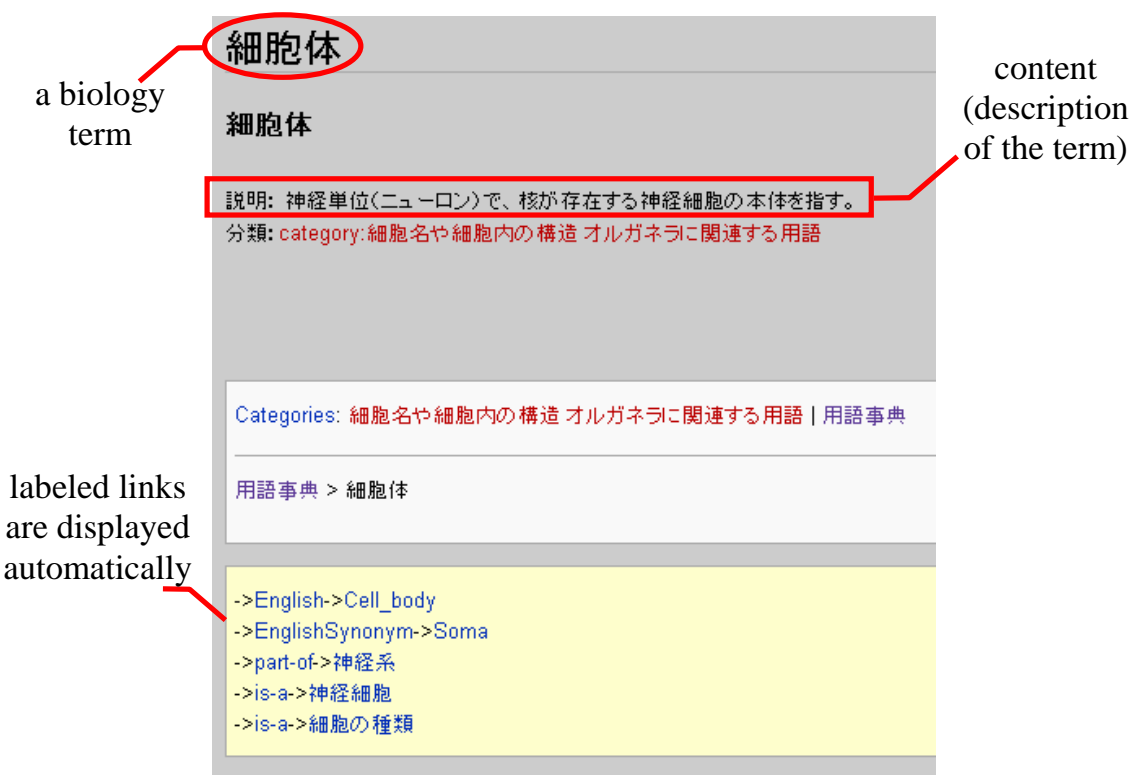

Fig. 6. Wiki page of a biology term in the Japanese Biodictionary

\subsection{Semantic Wiki for the development of a manufacturing feature library}

In a feature-based process planning system, a manufacturing feature library plays an important role for the extraction of manufacturing information for the generation of 
process plans [7]. However, manufacturing technologies are progressing, and manufacturing information used in a particular factory may not be the same as the other factory. It is necessary to enable the management of the manufacturing information flexibly. In other words, it is essential to build a manufacturing feature library that is easy to modify and to customize. Semantic Wiki provides the solution.

For the development of the manufacturing feature library, a manufacturing feature ontology is created as the structure of the library (see Fig.7). This lightweight ontology is created as follows. First, manufacturing features such as step, slot etc are listed up. The manufacturing features used in this research are based on the library proposed by CAM-I [8]. Sub-classes of these manufacturing features are created by describing the manufacturing methods to create the shape of the parent classes. Subclasses of these sub-classes are created by describing the tool types required for the manufacturing method. Instances of the lowest classes are created. Each instance contains specific manufacturing information, such as machine type, tool type, machining speed etc.

For the development of a Semantic Wiki-based manufacturing feature library, a new namespace ("MF:") is created. This namespace is used to handle manufacturing feature classes. Wiki syntax [[MF:feature_subclass|subclass]] is used to create class and sub-class relation of manufacturing features (see Fig.8). In the feature library, manufacturing information is also handled as Wiki pages. This allows the flexible management of manufacturing information, as well as the relation between the manufacturing feature's instance and the manufacturing information.

Semantic Wiki enables the creation of a structured manufacturing feature library. As data can be stored as RDF triples, the data can be processed by applications, which will support the automatic extraction of manufacturing information.

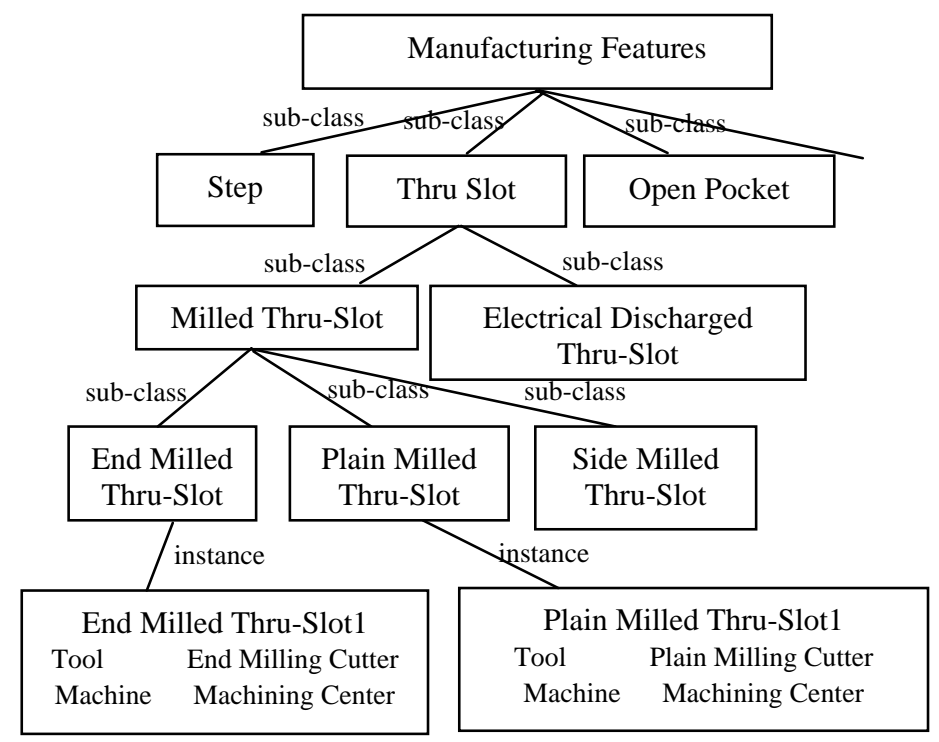

Fig. 7. A manufacturing feature ontology 


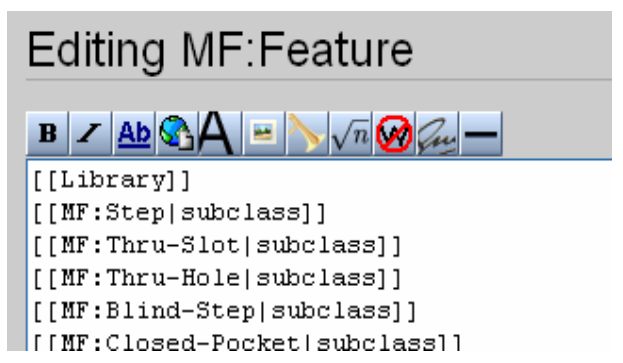

Fig. 8. Creating class and sub-class relation of the manufacturing features

\section{Conclusion}

The proposed Semantic Wiki is an extension of MediaWiki. It is able to write labeled links to construct RDF triples. It is a very simple software and as one tries to use this software, one may enjoy a visible editing of Wiki pages' relations. As it also inherits all the functions available in MediaWiki, it is a useful tool for the collaborative editing of contents and metadata according to simple RDF statements.

The implementation cases of the proposed Semantic Wiki show that it is an easyto-use lightweight knowledge management system.

\section{References}

1. Leuf, B., Cunningham, W.: The Wiki Way: Quick Collaboration on the Web. AddisonWesley, Boston (2001)

2. Wagner, C.: Wiki: a Technology for Conversational Knowledge Management and Group Collaboration. Communications of the Association for Information Systems, 13 (2004) 265-289

3. Raman, M., Ryan, T., Olfman, L.: Designing Knowledge Management Systems for Teaching and Learning with Wiki Technology, Journal of Information Systems Education, 16 (2005) 311-320

4. Cortese, A.: Business is Toying with a Web Tool. The New York Times, May 19 (2003)

5. Lassila, O.: Web Metadata: A Matter of Semantics, IEEE Internet Computing, Vol. 2, No. 4, (1998) 30-37

6. Takeda, H., Muljadi, H.: Towards Semantic MediaWiki. In Proc. of the 9th Semantic Web and Ontology SIG, Japanese Society for Artificial Intelligence. (2005) (in Japanese)

7. Ando, K., Muljadi, H., Takeda, H., Ogawa, M.: Development of Feature Library for a Process Planning System. In Proc. of the 8th Int'l Conf. on Manufacturing \& Management. (2004) 885-890

8. Butterfield, W.R., Green, M.K., Scott, D.C., Stoker, W.J.: Part Features for Process Planning. Computer Aided Manufacturing International (CAM-I), Document R-86-PPP-01 (1988) 